\title{
207 - Cognitive deficits in specific settings: challenges for the assessment and disease management
}

General synopsis of the Symposium: Cognitive impairment is an important geriatric syndrome. As the global population ages exponentially, the prevalence of cognitive impairment and dementia is increasing, and mental status assessment is key in the evaluation of older adults. However, there are several pitfalls during cognitive testing and special clinical situations that demands a more in-depth knowledge and attention from the examiner. The overall objective of this symposium is to show strategies and particularities of cognitive assessment in different settings (in the clinic, inside the hospital) and clinical scenarios (early onset impairment, mental illness comorbidity).

\section{Oral Communication 1: Cognitive Impairment in Younger People: Needs and Service Developments (ELIZABETA MUKAETOVA-LADINSKA ${ }^{1,2}$; Matthew Critchfield $^{2}$ )}

1 - Department of Neuroscience, Psychology and Behaviour, University of Leicester, Leicester; 2 - Younger Onset Dementia Assessment Service (YODAS), Leicestershire Partnership NHS Trust, Leicester, UK

Younger people with subjective memory problems referred to our specialist memory services represent a heterogeneous group. Only a small portion of them will have an early onset dementia of the Alzheimer's Disease, or their memory pro0blems be a result of cerebrovascular disease. We will address the latest NICE guidelines for dementia, as well as the Royal College report for younger people with dementia, and in particular:

- Referral triage to memory clinic

- Clinical dementia assessments for younger people

- Most frequent dementia diagnosis in this age group

- Role of Multidisciplinary Team in the diagnostic dementia pathway and post diagnostic support

- Long-term management of cognitive deficits in younger people

- Need of specialist young onset dementia services

This communication will explore different clinical scenarios generated from a real clinical setting. The attendees will be stimulated to knowledge exchange among both clinical and nonclinical specialists working in different clinical settings and exchange working experiences. We aim to contribute towards better understanding of challenges faced by both service users and clinicians working in the young onset dementia services.

Oral Communication 2: Cognitive impairment in acutely-ill medical patients (JOAQUIM CEREJEIRA, Portugal ${ }^{1,2,3}$ )

1- Center for Neuroscience and Cell Biology, University of Coimbra

2- Faculty of Medicine, University of Coimbra

3- Psychiatry service, Coimbra University Hospitals

Acutely medically ill older patients are at risk of having, by the time of their hospitalization, any kind of cognitive impairment. Delirium is a very common problem in acute hospital geriatric settings, with a point prevalence of about $24 \%$, and this condition is associated with extremely adverse outcomes including long term worsening of cognitive function and higher rates of progression to dementia. Dementia may already be present in patients hospitalized in acute medical settings, and delirium and dementia may coexist. These are complex conditions characterized by non-linear interactions among etiological distinct factors and by graded and continuous changes in cognitive, behavioral and emotional 
symptoms. The presence of delirium superimposed to dementia may significantly increase the rate of mortality and loss of independence. These syndromes are often overlooked by health care professionals and this failure to recognize and appropriately assess and manage the health of individuals facing these conditions can lead to substandard care and worse outcomes during hospital stay. However, despite of the clear need for a proper diagnosis and management of these conditions in acutely ill older adults, its correct diagnosis may prove to be difficult. Difficulties in cognitive assessment of these patients, mainly due to their poor status and communication abilities while hospitalized is a challenge for clinicians who are faced with these patients. There is a need to discuss and unravel new methods to adequately diagnose these individuals while in-treatment in order to provide the consequent interventions to reduce the expected adverse outcomes of these conditions after discharge. TO sum, a deeper understanding on how to assess, manage, and follow up geriatric patients with cognitive impairment in acute hospital settings is vital to improve their care and eventually to prevent the worsening of their cognitive status.

\section{Oral Communication 3: Cognitive impairment in the clinic- beyond MMSE and MOCA (IVAN APRAHAMIAN, Brazil ${ }^{1}$ )}

1- Faculty of Medicine of Jundiaí, Jundiaí, Brazil

The aging of societies is a global phenomenon and more geriatric syndromes are becoming frequent in the clinic. Among those, one of utmost importance is cognitive impairment. Sociodemographic projections estimate that there will be 135 million people with dementia in 2050, and 71\% of whom will live in low or middle-income countries. Unfortunately, misdiagnosis rates of the disease are high even in high-income countries. Early detection of cognitive impairment can provide treatment for reversible causes, improve quality of life, and reduce costs. Quick and simple cognitive screening tests should be the initial evaluation of older patients with suspected cognitive impairment in the clinic. Currently, the Mini-Mental State Examination (MMSE) or the Montreal Cognitive Assessment (MoCA) are among the most commonly used tests recommended for this purpose in clinical practice. However, these instruments are requiring payment for use and sometimes biased by frequent situations such as the subject's educational level, age, socioeconomic status and symptoms of depression. Moreover, some sub-items of these tests may contribute variably to their total score. Thus, alternative instruments and in-depth knowledge of cognitive assessment is fundamental to the clinician that delivers geriatric care.

\section{Oral Communication 4: Cognitive impairment in schizophrenia: how age affects neuropsychological} assessment? (ANA RITA SILVA, Coimbra, Portugal ${ }^{1,2}$ )

1- Center for Neuroscience and Cell Biology, University of Coimbra

2- Center for Research in Neuropsychology and Cognitive Behavioral Interventions

- CINEICC, University of Coimbra

Abstract: Cognitive deficits are consistently being described as the core feature of schizophrenia, with executive functions being the most affected function (Green et al., 2000; Harvey, 2001). Cognitive impairment has been described having a significant influence in patients' low levels of recovery and low rehabilitation success. As schizophrenia patients get old, an age-related cognitive impairment is added to the disease cognitive deficits, thus possibly reducing the positive effects of treatment if the individuals already had poor cognitive performance after theirs first psychotic episode (Mosiolek et al., 2016). The extent to which aging increases significantly the burden of cognitive deficits in these patients remains to be clarified. Some suggest that people with schizophrenia follow a similar age-related trajectory of cognitive decline as healthy individuals (Valsdottir et al., 2019), with their cognitive impairment more sever as their age being more related with the higher prevalence of positive symptoms and the extent of cognitive impairment at the onset of the first psychotic symptoms. 
However, the recent evidence for the changes in brain networks affected over the lifespan of schizophrenia patients makes It relevant to understand which neuropsychological measures should be used as sensitive markers of those changes and that can discriminate between age-related changes and schizophrenia associated changes in cognition. We will describe some longitudinal studies and casecontrol studies that will allow us to describe a set of tools that could be used in clinical practice by neuropsychologists to support a proper cognitive assessment of these patients. 